Pacific Journal of Mathematics

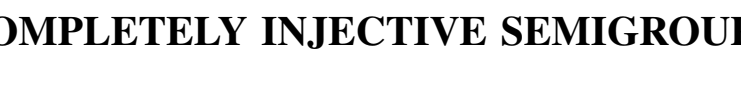




\title{
COMPLETELY INJECTIVE SEMIGROUPS
}

\author{
E. H. Feller and R. L. Gantos
}

A semigroup $S$ with identity is termed completely right injective if every right unitary $S$-system is injective. The semigroup $S$ is called completely injective if every right and left unitary $S$-system is injective. We prove that $S$ is completely injective if and only if $S$ is a semigroup with zero, where every right ideal and every left ideal of $S$ is generated by an idempotent. This condition is equivalent to the statement that $S$ is an inverse semigroup with zero, whose idempotents are dually well-ordered.

If $S$ is completely injective and if $e$ is an idempotent in $S$, then $e S e$, and every two-sided ideal of $S$, is completely injective.

A completely injective semigroup $S$ is termed central if $S$ is the union of groups. If $S$ is completely injective and $S$ has a finite number of right ideals, or if the two-sided ideals of $S$ are local, then $S$ is central.

2. Main theorems. Throughout this paper $S$ will always denote a semigroup with 1 , and all S-systems will be unitary. The set of idempotents of any semigroup $T$ will be denoted by $E(T)$.

Using 2.2, and the proof of 2.7 of [3], we have the first part of the following theorem.

THEOREM 2.1. If $S$ is completely right injective, then every right ideal is generated by an idempotent. Thus the right ideals form a chain under set inclusion, which is dually well-ordered. In addition, $S$ contains a zero element.

Proof. From the proof of 2.6 of [3], we have $S$ contains a left zero 0 . Now $0 S=\{0\}$ is contained in every right ideal. Hence for $a \in S$, then $0 S \subseteq a 0 S$. Thus $0=a 0 x=a 0$.

Lemma 2.2. Let $e, f \in E(S)$.

(i) If every right ideal of $S$ is generated by an idempotent, then $S e \subseteq S f$ implies $e S \subseteq f S$.

(ii) If every left ideal of $S$ is generated by an idempotent, then $e S \subseteq f S$ implies $S e \subseteq S f$.

(iii) If every right and left ideal of $S$ is generated by an idempotent, then $S e \subseteq S f$ if and only if $e S \subseteq f S$. In particular, Se $=S f$ if and only if $e S=f S$. 
Proof. Clearly, $e S \subseteq f S$ if and only if $f e=e$, and $S e \subseteq S f$ if and only if $e f=e$. To prove (i), suppose $S e \subseteq S f$. Then $e f=e$. Either $e S \subseteq f S$ or $f S \subset e S$. The latter is impossible for then $f \neq e$ and $e f=f$.

Part (ii) is proved in a similar way, while (iii) follows from (i) and (ii).

LEMMA 2.3. If every right and left ideal of $S$ is generated by an idempotent, then $S$ is an inverse semigroup. Moreover, $E(S)$ is a chain under the natural partial ordering, which is dually wellordered.

Proof. The fact that $S$ is regular follows from Lemma 1.13 of $[1$, p. 27]. We shall now prove (i) of Lemma 1.17 of $[1$, p. 28] to show that $S$ is inverse.

For $e, f \in E(S)$, then either $e S \subseteq f S$ or $f S \subseteq e S$. If $e S \subseteq f S$, then by 2.2 we have $S e \leqq S f$. Hence $f e=e f=e$, and $e \leqq f$ under the natural partial ordering. Thus the idempotents of $S$ commute, and form a chain.

Since $E(S)$ is commutative, then $e \leqq f$ if and only if $e S \subseteq f S$. By 2.1, the dual well-ordering of the right ideals implies that any nonempty subset $\left\{e_{\alpha} \mid \alpha \in I\right\}$ of $E(S)$ contains a greatest element; namely the idempotent which generates $\bigcup_{\alpha \in I} e_{\alpha} S$.

LEMMA 2.4. If $T$ is an inverse semigroup and $e \in E(T)$, then aea $\alpha^{-1}$ and $\alpha^{-1} e a$ are in $E(T)$.

Proof. Since $a^{-1} a \in E(T)$ and $E(T)$ is commutative, then

$$
\left(a e a^{-1}\right)\left(a e a^{-1}\right)=a\left(a^{-1} a\right) e^{2} a^{-1}=a e a^{-1} .
$$

Similarly, $\left(a^{-1} e a\right)^{2}=a^{-1} e a$.

FIRst MAIN TheORem 2.5. A semigroup $S$ is completely injective if and only if $S$ is a semigroup with zero, and every left and right ideal of $S$ is generated by an idempotent.

Proof. From 2.1, we have the "only if" part of this theorem.

Suppose now $S$ is a semigroup with zero, and every right and left ideal is generated by an idempotent. From 2.3, $S$ is an inverse semigroup and $E(S)$ is dually well-ordered. Using the same technique employed in the proof of Theorem 2.6 of [3], we show every right $S$-system is injective. A similar argument shows left $S$-systems are injective.

Let $M, P$, and $R$ be $S$-systems where $P \subseteq R$. If $f: P \rightarrow M$ is a 
$S$-homomorphism of $P_{S}$ into $M_{S}$, let $\left(P_{0}, f_{0}\right)$ be the maximal pair defined in the proof of 2.6 of [3]. To show $M$ is injective, it suffices to show $P_{0}=R$. Suppose $r \in R, r \notin P$, and let $A=\left\{a \in S \mid r a \in P_{0}\right\}$. As in 2.6 of [3], we will reach a contradiction for $P_{0} \neq R$, if we can show the existence of an $S$-homomorphism $h: r S \rightarrow M$ which agrees with $f_{0}$ on $P_{0} \cap r S$. If $A$ is empty, the argument is the same as in 2.6 of [3].

Suppose $A$ is nonempty. Then $A=e S$, for $e \in E(S)$. Let $h$ be the same mapping, $h(r s)=z e s$ for all $s \in S$, defined in 2.6 of [3]. We need only show that $h$ is single-valued. The argument of 2.6 of [3] will then complete the proof.

As shown in 2.6 of [3], $h$ will be single-valued if and only if $r s_{1}=r s_{2}$ implies $r e s_{1}=r e s_{2}$, for all $s_{1}, s_{2} \in S$. Since $S$ is inverse, then $r e s_{1}=r\left(e s_{1} s_{1}^{-1} s_{1}\right)=r\left(s_{1} s_{1}^{-1}\right) e s_{1}=\left(r s_{1}\right) s_{1}^{-1} e s_{1}=r s_{2} s_{1}^{-1} e s_{1}$. Likewise res $s_{2}=$ $r s_{1} s_{2}^{-1} e s_{2}$. Since $e s_{1}$, and $e s_{2}$ belong to $A$, then $r e s_{1}$ and $r e s_{2}$ belong to $P_{0}$. Therefore $s_{2} s_{1}^{-1} e s_{1}$ and $s_{1} s_{2}^{-1} e s_{2}$ belong to $A$. Since $A=e S$, then $s_{2} s_{1}^{-1} e s_{1}=$ $e s_{2} s_{1}^{-1} e s_{1} ; \quad$ consequently $r e s_{1}=r e s_{2} s_{1}^{-1} e s$. Likewise res $=r e s_{1} s_{2}^{-1} e s_{2}$. Using the fact that idempotents commute and Lemma 2.4, we have

$$
\begin{aligned}
r e s_{1} & =\left(r e s_{2}\right) s_{1}^{-1} e s_{1}=\left(r e s_{1} s_{2}^{-1} e s_{1}\right) s_{1}^{-1} e s_{1} \\
& =r e s_{1}\left(s_{2}^{-1} e s_{2}\right)\left(s_{1}^{-1} e s_{1}\right)=r e s_{1}\left(s_{1}^{-1} e s_{1}\right)\left(s_{2}^{-1} e s_{2}\right) \\
& =r\left(e s_{1} s_{1}^{-1} e s_{1}\right) s_{2}^{-1} e s_{2}=r e s_{1} s_{2}^{-1} e s_{2}=r e s_{2} .
\end{aligned}
$$

Second MaIn Theorem 2.6. A semigroup $T$ is completely injective if and only if $T$ is an inverse semigroup with zero, and $E(T)$ is dually well-ordered.

Proof. The definition of completely injective implies such semigroups contain an identity 1 . Using 2.5 and 2.3 , we have the necessity.

Conversely, suppose $T$ is inverse with zero and $E(T)$ is dually well-ordered. Using the argument in the proof of Lemma 2.1 of [4], the greatest element of $E(T)$ is the identity element of $T$.

Let $R$ be any right ideal of $T$. By Theorem 1.13 of [1, p. 27], the principal right ideals of $T$ are generated by idempotents. Therefore $E(T) \cap R$ is not empty. Since $E(T)$ is dually well-ordered, then $E(T) \cap R$ contains a greatest element $f$. It follows $R=f T$. In this way every right and left ideal is generated by an idempotent. Applying 2.5 we have $T$ is completely injective.

If $S$ is completely injective, it is of interest to note that the $\mathscr{R}$-classes of $S$, defined in [1, p. 47], are of the form $e S \backslash f S$, where $f S$ is maximal in eS.

Example 2.7. N. R. Reilly [4] called a semigroup $T$ an $\omega$-semi- 
group if and only if there exists a one-to-one may $\varphi$ of $E(T)$, which is commutative, onto the set of nonnegative integers such that

$$
\varphi(e) \leqq \varphi(f)
$$

if and only if $f \leqq e$. Thus $E(T)$ is dually well-ordered. Applying 2.6, for any inverse $\omega$-semigroup $T$, we have $T^{0}=T \cup 0$ is completely injective. The bisimple $\omega$-semigroups are concrete examples of inverse $\omega$-semigroups. In particular, the bicyclic semigroup of $[1, p .43]$ with zero adjoined is completely injective. These provide examples of completely injective semigroups, which are not the union of groups, as discussed in [3].

A trivial example of a completely right injective semigroup which is not completely left injective is a right zero semigroup containing: two or more elements with 0 and 1 adjoined. In fact, applying the technique of 2.5, the authors have shown that if $S$ is a right 0 -simple semigroup containing an idempotent $e \neq 0$, then $S^{1}=S \cup 1$ is completely right injective.

3. Properties of completely injective semigroups. In §'s 3 and 4, $S$ will always denote a completely injective semigroup. We begin this section with a discussion of a one-to-one correspondence between the lattices of right ideals and of left kernel congruences belonging to $S$-endomorphisms of ${ }_{S} S$. The left kernel congruence belonging to a $S$-endomorphism $g$ of ${ }_{S} S$ is defined to be that left congruence $\rho$ on $S$ given by $a \rho b$ if and only if $g(a)=g(b)$.

Definition 3.1. If $K$ is a subset of $S$, let $\rho(K)[\lambda(K)]$ denote the right [left] congruence of $S$ defined by: $(a, b) \in \rho(K)[(a, b) \in \lambda(K)]$ if and only if $k a=k b[a k=b k]$ for all $k \in K$. If $\sigma$ is a right [left] congruence on $S$, let $\ell(\sigma)[z(\sigma)$ ] denote the set of all $s \in S$ such that if $a \sigma b$, then $s a=s b[a s=b s]$. Clearly, $\ell(\sigma)[z(\sigma)]$ is a left [right] ideal of $S$.

Proposition 3.2. If $e \in E(S)$, then $\leadsto(\lambda(e S))=e S$ and $\ell(\rho(S e))=S e$.

Proof. If $b \in r(\lambda(e S))$, then $\lambda(e) \leqq \lambda(b)$. Thus the mapping $g: x e \rightarrow$ $x b$ is an $S$-homomorphism of $S e$ onto $S b$. Now $b=g(e)=e g(e)$. Therefore $b \in e S$ and $:(\lambda(e S)) \subseteq e S$. Since the opposite inclusion is immediate, we have equality. Similarly, $\ell(\rho(S e))=S e$.

The left congruence $\lambda(e S)$, wehere $e \in E(S)$, is the left kernel congruence belonging to the $S$-endomorphism $h:{ }_{S} S \rightarrow{ }_{S} S$, where $h(x)=$ $x e$ for all $x \in S$. Conversely, every left kernel congruence belonging to a $S$-endomorphism $h$ of ${ }_{s} S$ is of this form. Indeed, the left kernel 
congruence belonging to $h$ is $\lambda(h(1) S)$, which equals $\lambda(e S)$ for some $e \in E(S)$.

Since $e_{1} S \subseteq e_{2} S$ implies $\lambda\left(e_{1} S\right) \supseteqq \lambda\left(e_{2} S\right)$, then 3.2 implies that the mapping $e S \rightarrow \lambda(e S)$ is a one-to-one inclusion reversing correspondence between the lattice of right ideals of $S$ and the set $\mathscr{K}$ of all left kernel congruences belonging to $S$-endomorphisms of ${ }_{s} S$. Thus we have the following theorem.

Theorem 3.3. The lattice of right ideals of $S$ and the lattice of left kernel congruences belonging to $S$-endomorphisms of ${ }_{S} S$ are dual isomorphic.

Thus $S$ satisfies the minimum condition (D.C.C.) on right ideals if and only if $\mathscr{K}$ satisfies the maximum condition (A.C.C.). These results are similar to results for quasi-Frobenius rings.

Note that if $\sigma \in \mathscr{K}$, then $\lambda(z(\sigma))=\sigma$. It is not difficult to show this relation is not true for an arbitrary left congruence on $S$.

Next we show certain subsystems of $S$ are completely injective.

THEOREM 3.4. For every $e \in E(S)$, eSe is completely injective.

Proof. We show every left and right ideal of $e S e$ is generated by an idempotent. Let $L$ be a left ideal of $e S e$. It follows directly that $L=S L \cap e S e$. Now $S L=S f$, for some $f \in E(S)$. Using Lemma 1.19 of $[1$, p. 30], we have $L=S f \cap e S e=S f \cap S e \cap e S=S f e \cap e S=$ $(e S e) f$. If $e f=e$, then $L=e S e$. If $e f=f$, then $f=e f e \in e S e$, and $L=(e S e) f$. A similar argument holds for right ideals.

If $H$ is a two-sided ideal of $S$, we have by 2.2 and Theorem 1.17(ii) of [1, p. 28], that $H=e S=S e$. Hence $H=e S \cap S e=e S e$ and we can write

Corollary 3.5. Every two-sided ideal of $S$ is completely injective.

4. Central completely injective semigroups. Throughout this section, $S$ denotes a completely injective semigroup and $T$ an arbitrary semigroup. If $E(T)$ is contained in the center of $T$, then $T$ is termed central. In [3], the authors determined a structure for central completely injective semigroups. We use the fact that an inverse semigroup $T$ is central if and only if $T$ is the union of groups (see the proof of 2.8 of [3]). Applying this together with 2.6 we have 4.1 and 4.2.

THEOREM 4.1. A semigroup $T$ with 1 is central completely injective if and only if $T$ is an inverse semigroup with $0, E(T)$ is 
dually well-ordered, and $T$ is a union of groups.

THEOREM 4.2. $S$ is central if and only if $S$ is a union of groups.

Certainly, there are many conditions on an inverse semigroup which imply that it is the union of groups. For example, 7.4 of [2, p. 41] would imply that $S$ is central if and only if the left and right units of each element are equal.

Next we shall give a condition in terms of local semigroups. Using the terminology of [1, p. 21], if $T$ is a semigroup with 1 , then an element $a$ in $T$ is called a right [left] unit provided there exist $x \in T$ such that $a x=1[x a=1]$. A left and right unit is called a unit.

Proposition 4.3. A semigroup $T$ with 1 is termed local provided one of the following equivalent conditions are satisfied.

(i) Every right unit is a left unit.

(ii) The set of nonunits form a proper ideal of $T$.

(iii) $T$ contains an ideal, which is a unique maximal right ideal.

ThEOREM 4.4. $S$ is central if and only if the two-sided ideals of $S$ are local.

Proof. If $S$ is central, then each two-sided ideal of $S$ has the form $e S$, where the $f S$ of 2.11 of [3] satisfies (iii) of 4.3. Thus $e S$ is local.

To prove the converse we shall establish the statement, "all the idempotents of $S$ are contained in its center". For $S, E(S)$ is dually well-ordered. Thus we can list the elements of $E(S)$ as

$$
1=e_{0}>e_{1}>e_{2} \cdots>e_{\alpha}>\cdots>0
$$

where the subscripts are ordinal numbers less than the ordinal number of $E(S)$. It follows

$$
S=e_{0} S \supset e_{1} S \supset e_{2} S \supset \cdots \supset e_{\alpha} S \supset \cdots \supset 0 .
$$

We use transfinite induction to prove the above statement. To show that $e_{1}$ is in the center of $S$, let $K$ be the set of nonunits of $S$. Since $S$ is local, then $K$ is a two-sided ideal which is a unique maximal right ideal of $S$. Thus $K=e_{1} S$ and, as in the discussion preceding 3.5, $e_{1} S=S e_{1}$. Hence $e_{1}$ is in the center of $S$.

Assume inductively that all $e_{\alpha}$, for $\alpha<\beta$, are in the center. If $\beta$ is not a limit ordinal, then $\beta=\alpha+1$, where $e_{\alpha}$ is in the center. Hence $e_{\alpha} S$ is local. Using the fact that a right ideal of an ideal of $S$ is itself a right ideal of $S$, then the argument in the preceding 
paragraph can be applied to show that $e_{\beta}$ is in the center.

If $e_{\beta}$ is a limit ordinal, then $\bigcap_{\alpha<\beta} e_{\alpha} S=e_{\beta} S$. Since the $e_{\alpha} S$, for $\alpha<\beta$, are two-sided ideals, then $e_{\beta} S$ is a two-sided ideal and $e_{\beta}$ is in the center.

One could use 4.2 to prove the following result. However, 4.5 follows directly from 7.5 of [2, p. 41]. The second part is a consequence of 3.3 .

Proposition 4.5. If $S$ satisfies the minimum condition for right ideals, or the maximum condition for left kernel congruences belonging to endomorphisms of ${ }_{S} S$, then $S$ is central.

A right $T$-system is projective if the usual diagram of right $T$ systems can be completed. We call a semigroup $T$ with identity 1 completely right projective if every right $T$-system is projective. In ring theory, completely projective is equivalent to completely injective. This is not the case for semigroups, which can be deduced from the following theorem.

THEOREM 4.6. If $T$ is a completely injective and completely right projective semigroup, then $T$ is a group with zero.

Proof. Let us denote the right annihilator of $x$ by $x^{*}$. For any idempotent $e$ of $T$, we have $e T \cap e^{*}=0$. Since the right ideals of $T$ form a chain, then $e^{*}=0$ for any nonzero $e$ in $E(T)$.

Let $N$ be a nonzero right ideal of $T$. Let $T / N$ denote the Rees factor $T$-system of $T$ by $N$ defined in [2, p. 252]. Let $g$ denote the natural homomorphism of $T$ onto $T / N$. Since $T / N$ is projective, there exists a monomorphism $h$ of $T / N$ into $T$ such that $g h=1$ and $h g$ is idempotent. Consequently $h g(1)=e, e \in E(T)$, and $h g(x)=e x$ for all $x \in T$.

By the definition of $g$, we have $h g(N)=h \overline{(0)}=0$. On the other hand, $h g(N)=h g(1) N=e N$. Thus $e N=0$. The discussion in the first paragraph together with the fact that $N \neq 0$ implies $e=0$. Hence $T / N=\overline{0}$, and $N=T$. Therefore each element in the semigroup of nonzero elements has a right inverse in $T$ and $T$ is a group with zero.

\section{BIBLIOGRAPHY}

1. A. H. Clifford and G. B. Preston, The algebraic theory of semigroups, Vol. I, Math. Surveys No. 7, Amer. Math. Soc., Providence, R. I., 1961.

2. - Vol. II, 1967. 
3. E. H. Feller and R. L. Gantos, Completely injective semigroups with central idem. potents, Glasgrow Math. J. (to appear)

4. N. R. Reilly, Bisimple w-semigroups, Proc. Glasgow Math. Assoc. 7 (1966), 160-169.

Received May 31, 1968. Research supported by the National Science Foundation under grant GP-6816 for the first author.

UNIVERSITY OF Wisconsin-MilwaUkeE 


\section{PACIFIC JOURNAL OF MATHEMATICS}

\section{EDITORS}

\author{
H. ROYDEN \\ Stanford University \\ Stanford, California \\ Richard Pierce \\ University of Washington \\ Seattle, Washington 98105
}

\author{
J. DugundJI \\ Department of Mathematics \\ University of Southern California \\ Los Angeles, California 90007 \\ BASIL GoRDON \\ University of California \\ Los Angeles, California 90024
}

\section{ASSOCIATE EDITORS}
E. F. BECKENBACH
B. H. Neumann
F. WOLF
K. YoSHIDA

\section{SUPPORTING INSTITUTIONS}

\author{
UNIVERSITY OF BRITISH COLUMBIA \\ CALIFORNIA INSTITUTE OF TECHNOLOGY \\ UNIVERSITY OF CALIFORNIA \\ MONTANA STATE UNIVERSITY \\ UNIVERSITY OF NEVADA \\ NEW MEXICO STATE UNIVERSITY \\ OREGON STATE UNIVERSITY \\ UNIVERSITY OF OREGON \\ OSAKA UNIVERSITY \\ UNIVERSITY OF SOUTHERN CALIFORNIA
}

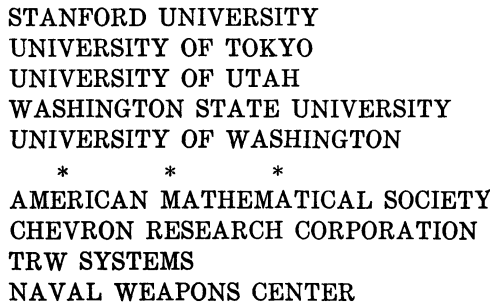

The Supporting Institutions listed above contribute to the cost of publication of this Journal, but they are not owners or publishers and have no responsibility for its content or policies.

Mathematical papers intended for publication in the Pacific Journal of Mathematics should be in typed form or offset-reproduced, double spaced with large margins. Underline Greek letters in red, German in green, and script in blue. The first paragraph or two must be capable of being used separately as a synopsis of the entire paper. It should not contain references to the bibliography. Manuscripts, in duplicate if possible, may be sent to any one of the four editors. Please classify according to the scheme of Math. Rev. 36, 1539-1546. All other communications to the editors should be addressed to the managing editor, Richard Arens, University of California, Los Angeles, California, 90024.

50 reprints are provided free for each article; additional copies may be obtained at cost in multiples of 50 .

The Pacific Journal of Mathematics is published monthly. Effective with Volume 16 the price per volume (3 numbers) is $\$ 8.00$; single issues, $\$ 3.00$. Special price for current issues to individual faculty members of supporting institutions and to individual members of the American Mathematical Society: $\$ 4.00$ per volume; single issues $\$ 1.50$. Back numbers are available.

Subscriptions, orders for back numbers, and changes of address should be sent to Pacific Journal of Mathematics, 103 Highland Boulevard, Berkeley, California, 94708.

PUBLISHED BY PACIFIC JOURNAL OF MATHEMATICS, A NON-PROFIT CORPORATION

Printed at Kokusai Bunken Insatsusha (International Academic Printing Co., Ltd.), 7-17, Fujimi 2-chome, Chiyoda-ku, Tokyo, Japan. 


\section{Pacific Journal of Mathematics}

\section{Vol. 31, No. $2 \quad$ December, 1969}

Efraim Pacillas Armendariz, Quasi-injective modules and stable torsion

classes..........................................

J. Adrian (John) Bondy, On Ulam's conjecture for separable graphs...

Vasily Cateforis and Francis Louis Sandomierski, On commutative rings over which the singular submodule is a direct summand for every module .....

Rafael Van Severen Chacon, Approximation of transformations with continuous

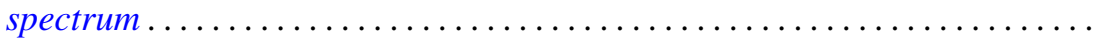

Raymond Frank Dickman and Alan Zame, Functionally compact spaces ...... 303

Ronald George Douglas and Walter Rudin, Approximation by inner

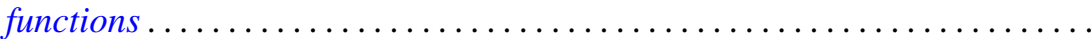

John Walter Duke, A note on the similarity of matrix and its conjugate

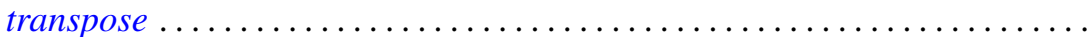

Micheal Neal Dyer and Allan John Sieradski, Coverings of mapping

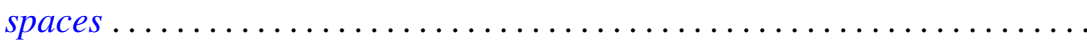

Donald Campbell Dykes, Weakly hypercentral subgroups of finite groups .....

Nancy Dykes, Mappings and realcompact spaces.....................

Edmund H. Feller and Richard Laham Gantos, Completely injective

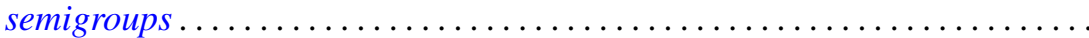

Irving Leonard Glicksberg, Semi-square-summable Fourier-Stieltjes

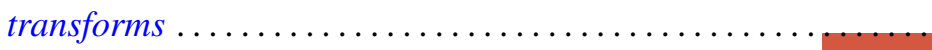

Samuel Irving Goldberg and Kentaro Yano, Integrability of almost cosymplectic structures...

Seymour Haber and Charles Freeman Osgood, On the sum $\sum\langle n \alpha\rangle^{-t}$ and numerical integration ..........................

Sav Roman Harasymiv, Dilations of rapidly decreasing functions ....

William Leonard Harkness and R. Shantaram, Convergence of a sequence of

transformations of distribution functions

Herbert Frederick Kreimer, Jr., A note on the outer Galois theory of rings ...

James Donald Kuelbs, Abstract Wiener spaces and applications to analysis. .

Roland Edwin Larson, Minimal $T_{0}$-spaces and minimal $T_{D}$-spaces...

A. Meir and Ambikeshwar Sharma, On Ilyeff's conjecture .

Isaac Namioka and Robert Ralph Phelps, Tensor products of compact convex sets....

James L. Rovnyak, On the theory of unbounded Toeplitz operators ....

Benjamin L. Schwartz, Infinite self-interchange graphs.......

George Szeto, On the Brauer splitting theorem...

Takayuki Tamura, Semigroups satisfying identity $x y=f(x$,

Kenneth Tolo, Factorizable semigroups .................. 